\title{
Lasting Impact of Regret and Gratification on Resting Brain Activity and Its Relation to Depressive Traits
}

\author{
Hamdi Eryilmaz, ${ }^{1,2}$ Dimitri Van De Ville, ${ }^{2,3,4}$ Sophie Schwartz, ${ }^{1,2,5}$ and Patrik Vuilleumier ${ }^{1,2,5}$ \\ ${ }^{1}$ Laboratory of Neurology and Imaging of Cognition, Department of Neuroscience, University Medical School of Geneva, and ${ }^{2}$ Geneva Neuroscience Center, \\ University of Geneva, 1205 Geneva, Switzerland, ${ }^{3}$ Institute of Bioengineering, Ecole Polytechnique Fédérale de Lausanne, 1015 Lausanne, Switzerland, \\ ${ }^{4}$ Department of Radiology and Medical Informatics, University of Geneva, 1205 Geneva, Switzerland, and ${ }^{5}$ Swiss Center for Affective Sciences, University of \\ Geneva, 1211 Geneva, Switzerland
}

Obtaining lower gains than rejected alternatives during decision making evokes feelings of regret, whereas higher gains elicit gratification. Although decision-related emotions produce lingering effects on mental state, neuroscience research has generally focused on transient brain responses to positive or negative events, but ignored more sustained consequences of emotional episodes on subsequent brain states. We investigated how spontaneous brain activity and functional connectivity at rest are modulated by postdecision regret and gratification in 18 healthy human subjects using a gambling task in fMRI. Differences between obtained and unobtained outcomes were manipulated parametrically to evoke different levels of regret or gratification. We investigated how individual personality traits related to depression and rumination affected these responses. Medial and ventral prefrontal areas differentially responded to favorable and unfavorable outcomes during the gambling period. More critically, during subsequent rest, rostral anterior and posterior cingulate cortex, ventral striatum, and insula showed parametric response to the gratification level of preceding outcomes. Functional coupling of posterior cingulate with striatum and amygdala was also enhanced during rest after high gratification. Regret produced distinct changes in connectivity of subgenual cingulate with orbitofrontal cortex and thalamus. Interestingly, individual differences in depressive traits and ruminations correlated with activity of the striatum after gratification and orbitofrontal cortex after regret, respectively. By revealing lingering effects of decision-related emotions on key nodes of resting state networks, our findings illuminate how such emotions may influence self-reflective processing and subsequent behavioral adjustment, but also highlight the malleability of resting networks in emotional contexts.

Key words: cingulate; counterfactual emotions; fMRI; resting state; rumination; ventral striatum

\section{Introduction}

Earning or missing a reward may produce immediate emotions such as elation or disappointment, respectively. However, intense feelings are not restricted to acute responses to positive or negative events, but also entail more prolonged affective changes (Brosschot et al., 2010; Eryilmaz et al., 2011; Curci et al., 2013). Whereas emotions are characterized by transient episodes, more sustained affective states mediate mood and motivation processes (Scherer, 2005). Good news often makes us feel cheerful for the remainder of the day, whereas we may brood bitterly and

\footnotetext{
Received Jan. 6, 2014; revised April 7, 2014; accepted April 29, 2014.

Author contributions: H.E., D.V.D.V., S.S., and P.V. designed research; H.E. performed research; H.E., D.V.D.V., S.S., and P.V. contributed unpublished reagents/analytic tools; H.E., S.S., and P.V. analyzed data; H.E., D.V.D.V., S.S., and P.V. wrote the paper.

This work was supported by the National Center of Competence in Research (NCCR) Affective Sciences financed by the Swiss National Science Foundation (Grant 51NF40-104897) and hosted by the University of Geneva, the Swiss National Science Foundation (Individual Project Grants 320030-135653 to S.S. and PP00P2-146318 to D.V.D.V.), and the Academic Society of Geneva (Foremane Fund to P.V.). We thank David Sander and Klaus Scherer for helpful discussions about the study design and interpretations.

The authors declare no competing financial interests.

Correspondence should be addressed to Hamdi Eryilmaz, Department of Psychiatry, Martinos Imaging Center, Massachusetts General Hospital, CNY 149, $13^{\text {th }}$ Street, Boston, MA 02129. E-mail: hamdi.eryilmaz@gmail.com.

DOI:10.1523/JNEUROSCI.0065-14.2014

Copyright $\odot 2014$ the authors $\quad 0270-6474 / 14 / 347825-11 \$ 15.00 / 0$
}

durably over a loss. Despite abundant research on brain response to various emotional stimuli and rewards (Armony and Vuilleumier, 2013), these more prolonged affective states remain understudied.

Lingering emotions frequently follow outcomes of our choices, particularly when we realize that another choice would have yielded better or worse outcomes (Mellers et al., 1999). This weighting of "what is" against "what might have been" is termed counterfactual processing (Mellers et al., 1999; Connolly and Butler, 2006) and constitutes a determinant feature of selfreferential emotions such as "regret." Unlike disappointment or sadness, regret implies a dimension of self-responsibility $(\mathrm{Ca}-$ mille et al., 2004; Coricelli et al., 2005; Summers and Duxbury, 2012). Conversely, obtaining the most desirable outcome from one's choice triggers "gratification" (Ortony et al., 1990; Connolly and Butler, 2006; Summers and Duxbury, 2012). Both regret and gratification produce long-lasting emotions with profound impacts on behavioral adjustment and motivation (Markman and Miller, 2006).

Here, we used fMRI to investigate the lingering influences of postdecision regret and gratification on brain activity at rest. Transient emotions (e.g., induced by movies) can produce longlasting effects on subsequent activity of the default mode network 


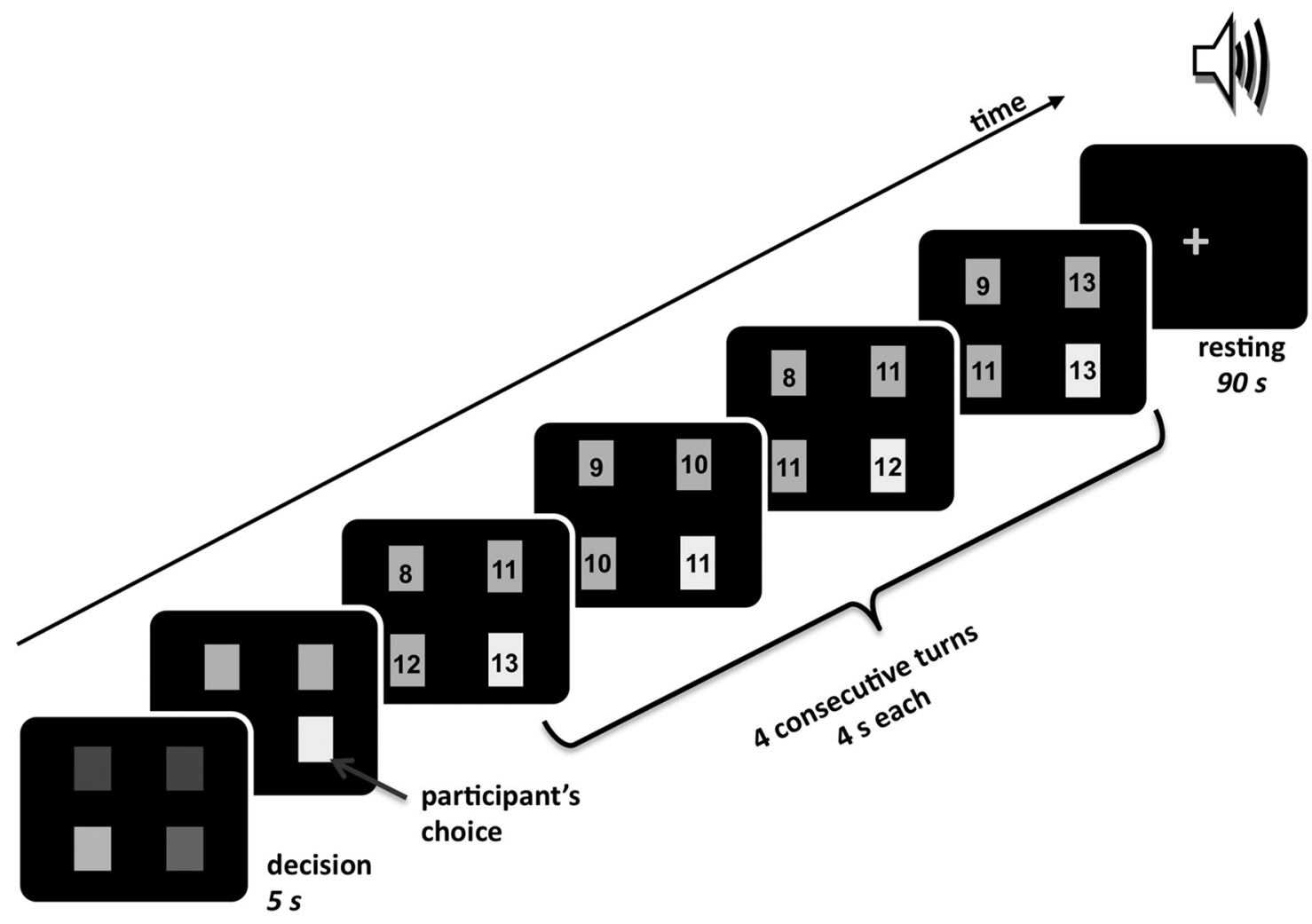

Figure 1. Illustration of the experimental paradigm. Each cycle started with a choice display (5 s) in which 4 colorful squares were presented on the screen, each with different and rapidly changing colors. Participants had to select 1 of these boxes by pressing on a keypad with 4 buttons. This started a "game block" with 4 sequential turns, each composed of a brief animation with flickering numbers in each box ( $2 \mathrm{~s}$ ) followed by outcome delivery ( $4 \mathrm{~s}$ ). The outcome screen displayed the number of points (from 1 to 23 ) offered by each box, with the participant's choice depicted in yellow and the height of boxes proportional to this amount to facilitate reading. The gain assigned to each box was manipulated to create 6 different outcome levels, such that the 4 turns could include either "favorable streaks" in which the chosen box yielded higher gains (with levels 1, 2, or 3 corresponding to decreasing differences relative to the unchosen boxes), or "unfavorable streaks," in which the chosen box yielded lower gains (with levels 4,5 , and 6 corresponding to increasing differences relative to the unchosen boxes). The "game block" was followed by a rest period ( $90 \mathrm{~s}$ ) during which the participants were instructed to let their mind wander with eyes closed. At the end of resting, a beep prompted them to open their eyes again and the experiment continued with a new choice and game block.

(DMN) and limbic areas at rest (Eryilmaz et al., 2011). A few fMRI studies investigated self-referential emotions such as regret (Coricelli et al., 2005; Chandrasekhar et al., 2008), guilt (Wagner et al., 2011), pride (Takahashi et al., 2008; Simon-Thomas et al., 2012), or rejoice (Chandrasekhar et al., 2008), but all focused on event-related responses to emotion-eliciting stimuli or scenarios rather than more prolonged affective states. These studies reported activations in medial and ventral prefrontal areas associated with self-referential processing and emotion regulation (Phillips et al., 2003) as well as limbic areas such as insula, amygdala, and striatum.

In addition, we investigated whether any lasting effect of decision-based emotions on resting activity might depend on individual personality traits such as proneness to depression and rumination (Brosschot et al., 2010). Ruminations imply prolonged focus on negative outcomes and are associated with depressive tendencies (Smith and Alloy, 2009). Counterfactual thinking is also related to posttraumatic distress (El Leithy et al., 2006) and negative mood (Sanna et al., 1996; Markman and Miller, 2006).

To these aims, we designed a gambling task that engaged counterfactual thinking (adapted from Camille et al., 2004 and Coricelli et al., 2005). Participants could experience streaks of favorable or unfavorable outcomes during which their gain was respectively higher or lower than rejected alternatives. Our main interest concerned brain activity in rest periods subsequent to outcomes with high or low gain.

\section{Materials and Methods}

Participants. A first group of 18 right-handed volunteers (8 female, age 19-34 years, mean 24.4 years) with no history of neurological and psychiatric disorders participated in the fMRI study. Another group of 11 healthy volunteers ( 8 females, age $23-32$ years, mean 26.5 years) participated in a behavioral control study. All participants signed a consent form approved by the local ethics committee and were reimbursed for their participation.

Experimental procedure. Our paradigm consisted of 18 cycles, each of which included a decision stage, a game block, and a rest block (in this order). At the decision stage, participants selected one of four boxes on the screen (Fig. 1). This was followed by the game block, during which participants observed the gains offered by each box in four consecutive turns. Differences between gains for the chosen box and rejected alternatives were systematically manipulated along three levels (i.e., small, medium, or large difference), leading to different degrees of gratification (alternative gains lower than that of the participant, streak levels 1-3) and regret (alternative gains higher than that of the participant, streak levels 4-6). After the last of the four outcomes, participants were instructed to close their eyes and let their mind wander freely for $90 \mathrm{~s}$ (rest block). After $90 \mathrm{~s}$, a short beep prompted them to open their eyes again and this was followed by a new game cycle.

Gambling task. We adapted a gambling task used in previous studies (Camille et al., 2004; Coricelli et al., 2005). As shown in Figure 1, at the beginning of each cycle, participants were presented with four boxes. They were instructed to choose, within $5 \mathrm{~s}, 1$ of these boxes by pressing 1 of 4 buttons on a keypad. They were told that each of the boxes was associated with a different number of points, from relatively low to high, and that their goal was to find the box that would offer the highest gain in 


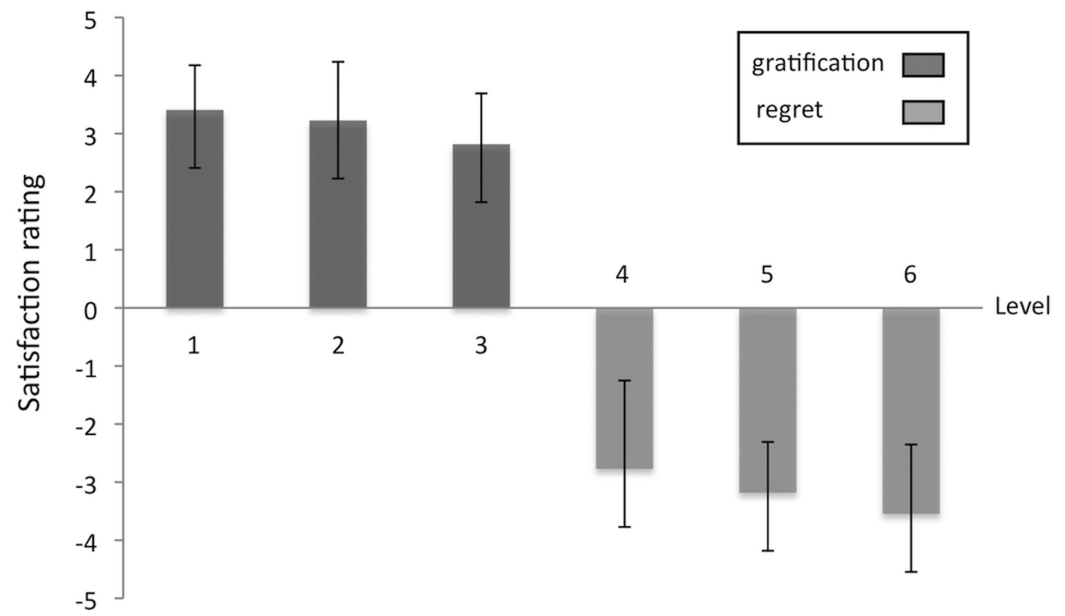

Figure 2. Results of the behavioral control study. Participants were asked to rate how satisfied they felt with their performance after a series of turns. Gratification and regret levels correspond to the difference between the obtained gain (balanced across different levels) and the unchosen options, decreasing from level 1 to 3 for favorable outcomes and increasing from level 4 to 6 for unfavorable outcomes. Satisfaction ratings were significantly modulated by this counterfactual difference in both cases; that is, when unchosen options were smaller (gratification) or greater (regret) than the actual gain. An ANOVA with factors actual gain (high, low) and alternative level $(1,2,3)$ revealed a significant interaction between the two factors $\left(F_{(2,20)}=3.60, p<0.046\right)$.

the subsequent game block. They were also told that the distribution of points among boxes changed in each game cycle and that their total score over the whole fMRI session would be proportionally transformed into a monetary bonus added to their reimbursement. There was no intermediate feedback about this total score, which was displayed only at the end of the scanning session. It was also made clear that the "best box" was determined by the computer for each new cycle and their task was only to make their best guess.

Once they had indicated their choice, participants observed the outcome that was revealed during the subsequent game block, which consisted of four consecutive turns. On each turn, a rapid succession of numbers was flickered in each of the boxes (for $2 \mathrm{~s}$ ) until a final static display showed the points attributed to all boxes on that turn (for $4 \mathrm{~s}$ ). As illustrated in Figure 1, the box chosen by the participant was highlighted in yellow and the size of each box varied with respect to the number of points to facilitate the reading of outcomes. A static outcome display ( $4 \mathrm{~s})$ allowed participants to observe the points they earned with the chosen box, but also to compare this gain with the points they would have earned with the rejected alternatives. This sequence of flickered numbers and outcomes assigned to each box was repeated in four successive turns after each choice made by the participants.

Importantly, the number of points and their distribution among boxes systematically varied from turn to turn but the boxes were programmed to give more or less similar amounts ( \pm 2 points) across all 4 turns within 1 game block (i.e., if the chosen box yielded a very high gain in one turn, it would also yield high points in the other 3 turns of the same block) to produce more or less similar differences relative to the unchosen boxes (i.e., all other boxes yielded higher or lower gains in the three successive turns for regret or gratification conditions, respectively). This allowed us to manipulate the overall subjective value of each game cycle, with some cycles leading participants to experience a series of more positive/rewarding outcomes and other cycles leading to a series of more negative/ disappointing outcomes.

This paradigm allowed us to induce either positive or negative emotions with a prolonged duration subsequent to free choices depending on both the gain that was obtained (chosen box) and the potential gains that were missed (unchosen boxes). Presumably, emotional reactions might be more intense for outcomes revealed in the first turn of a game block, but as the participants kept earning points (few or many) in the consecutive turns, these emotional reactions could actually build up and sustain until the end of the game block. Therefore, we expected that positive or negative affective states would be reliably evoked by favorable and unfavorable outcome streaks, respectively, and then also extend into the subsequent resting periods.

Crucially, even though we told participants that points were generated randomly by the computer as in a bandit machine, the distribution among boxes was programmed in a predetermined way. Previous research has shown that the knowledge of unobtained gains associated with rejected alternatives can strongly modulate subjective emotions evoked by a particular outcome such that the gain is rated as less positive if rejected alternatives would have led to a higher gain, but rated as more positive if rejected alternatives would have led to lower gain or losses, reflecting an impact of counterfactual thoughts on emotional appraisals (Mellers et al., 1997; Zeelenberg et al., 1998; Mellers et al., 1999; Camille et al., 2004; Coricelli et al., 2005). Here, we could induce gratification by assigning higher gains to chosen than to unchosen boxes or induce regret by assigning higher gains to unchosen boxes. In addition, we systematically manipulated the degree of gratification for high gains and the degree of regret for low gains by varying the difference in the number of points associated with the chosen and unchosen boxes. We designed six different streak levels corresponding to three degrees of gratification (for high gains) and three degrees of regret (for low gains). These six streak levels were pseudorandomly assigned to 18 game cycles (each with their corresponding rest blocks; i.e., there were three game and three rest periods for each of the six streak types). On average, the difference in gains between chosen and unchosen boxes was 11.7, 5.3, or 2.7 points for the favorable streaks (actual gain range 13-21), corresponding to large, medium, or small gratification levels (L1, L2, and L3, respectively) and $3.3,10.0$, and 14.7 points for the unfavorable streaks (actual gain range $2-8$ ), corresponding to small, medium, or large regret levels (L4, L5, and L6, respectively). These numbers were determined during pilot experiments (in other participants). Using these numbers, we were able to obtain an equal modulation of positive and negative emotion ratings for high gains (streak levels L1, L2, and L3) and low gains (streak levels L4, L5, and L6) as a function of the alternative outcomes associated with the unchosen boxes. This resulted in a balanced distribution of the gratification effects induced from the most (level 1) to the least (level 3) favorable streaks relative to the regret effects induced from the most (level 6) to the least (level 4) unfavorable streaks (i.e., creating comparable pairs of opposite emotions for levels 1-6, 2-5, and 3-4).

Our behavioral findings in a control study (see Results and Fig. 2) confirmed that this paradigm reliably produced a modulation of outcome ratings by the unobtained gains, as predicted by counterfactual processing. Debriefing after both the behavioral and fMRI experiments also verified that participants did not suspect these systematic manipulations.

MRI acquisition. Functional and structural MRI data were collected in a Siemens 3T Trio TIM system. EPI images were acquired in two continuous runs ( $\sim 20 \mathrm{~min}$ each $)$ with the following parameters: repetition time (TR) $1800 \mathrm{~ms}$, echo time (TE) $30 \mathrm{~ms}$, flip angle $80^{\circ}, 31$ axial slices, in-plane resolution of $3.2 \times 3.2 \mathrm{~mm}^{2}(64 \times 64$ matrix $)$, and slice thickness $3.84 \mathrm{~mm}$. We also acquired a T1-weighted anatomical image of each participant with parameters as follows: MPRAGE sequence, TR $1900 \mathrm{~ms}$, TE $2.32 \mathrm{~ms}$, flip angle $9^{\circ}, 192$ axial slices and isotropic voxel size of $0.90 \times$ $0.90 \times 0.90 \mathrm{~mm}^{3}(256 \times 256$ matrix $)$

Preprocessing of $f M R I$ data. All functional images were realigned to the first acquired image, corrected for slice timing, normalized to the MNI template (with $3 \times 3 \times 3 \mathrm{~mm}^{3}$ voxel size), and smoothed using a FWHM $=8 \mathrm{~mm}$ Gaussian kernel. Each participant's high-resolution anatomical image was also normalized to the MNI template with a $1 \times$ 
$1 \times 1 \mathrm{~mm}^{3}$ voxel size. All steps of preprocessing were performed using SPM8 (http://www.fil.ion.ucl.ac.uk/spm/).

GLM design. PMRI data were processed with SPM8 and submitted to a standard GLM analysis using parametric modulators that reflected the relative gain of the participant with respect to the rejected alternatives in a given turn. During game blocks, we defined a parametric modulator for every single turn, whereas during rest blocks, we used a parametric modulator representing the average relative gain over the preceding game block. This procedure provided us with a quantitative index for the degree of gratification and regret during both the game and the following rest period.

Our parametric modulator was the measure of how much more a participant gained relative to the rejected alternatives in a given turn. If the participant's gain is denoted by $\mathrm{p} 1$ and rejected alternatives by $\mathrm{p} 2, \mathrm{p} 3$, and $\mathrm{p} 4$, the parametric modulator for a single turn is calculated as follows:

$$
\mathrm{PM}_{\text {game }}=\mathrm{p} 1-(\mathrm{p} 2+\mathrm{p} 3+\mathrm{p} 4) / 3
$$

This parametric variable was computed for each turn in a game block. Each of the four successive turns within a cycle was modeled as a short block ( $4 \mathrm{~s}$ ) in the design matrix, with the addition of one parametric regressor for each turn in the gratification blocks (where participants gained more than the average of the rejected alternatives) and in the regret blocks (where participants gained less than the average of the rejected alternatives). The $90 \mathrm{~s}$ resting periods after pride and regret blocks were divided into three equal bins of $30 \mathrm{~s}$, which were entered separately into the design matrix (Eryilmaz et al., 2011); each again complemented with their corresponding parametric modulators representing gratification or regret levels in the preceding game block. Only one parametric variable was determined for each resting block by computing the average of the relative gain accumulated in successive turns during the preceding game block as follows:

$$
\mathrm{PM}_{\text {rest }}=\mathrm{p} 1_{\text {avg }}-\left(\mathrm{p} 2_{\mathrm{avg}}+\mathrm{p} 3_{\mathrm{avg}}+\mathrm{p} 4_{\mathrm{avg}}\right) / 3
$$

where $\mathrm{p} 1_{\text {avg }}$ is the participant's average gain for the chosen box in the preceding game block and p2-p4 the average of unobtained gains for rejected alternatives. We used the same parametric modulator value for all the three $30 \mathrm{~s}$ bins of rest after a given game block.

In total, our fMRI paradigm resulted in a design matrix composed of 16 regressors of interest (two game-related epochs for high or low gain conditions, their two corresponding parametric modulators for gratification or regret, the three postgratification and three postregret rest epochs, and their six corresponding parametric modulators for the different streak levels). We also inserted six realignment parameters to account for movement artifacts. All regressors were then submitted to a standard GLM analysis in SPM. Our design also accounted for any lowfrequency drift using a high-pass filter with cutoff frequency $1 / 256 \mathrm{~Hz}$. After parameter estimates and errors were computed for each regressor, these parameters were entered into a random-effects group analysis at the second level. We performed one-sample $t$ tests on the parametric contrasts to determine the effects of each outcome-related modulator on brain activity during both game and subsequent rest periods. For all parametric effects, we used a statistical threshold of $p=0.001$ (uncorrected) at the voxel level. All regions that survived this threshold were considered as activated and are reported.

In an additional analysis, we examined correlations with depressive traits. All participants filled out the following questionnaires: the Beck Depression Inventory (BDI) and the Rumination Response Score (RRS). Individual scores from each questionnaire were correlated with brain activity patterns using a second-level linear regression general linear model (GLM) on the main contrasts of interest, which were obtained using a first-level categorical design. The categorical design in the firstlevel GLM consisted of all six gratification/regret levels during game and their corresponding six rest blocks (divided into three bins of $30 \mathrm{~s}$, resulting in 18 regressors for rest). Response events corresponding to the choice of the box and six movement parameters were also added as nuisance regressors. For the second-level GLM, we used a statistical threshold of $p=0.001$ unless mentioned otherwise and reported all regions that survived this threshold.

Wavelet functional connectivity analysis. Interregional coupling of activity was assessed at predefined frequency sub-bands using wavelet decomposition (Richiardi et al., 2011; Eryilmaz et al., 2011). The wavelet transform provides a compact time-frequency representation of the time courses of brain activity. Here, we applied the nondecimated orthogonal cubic B-spline wavelet transform in the temporal domain. Details of the computation were described previously (Eryilmaz et al., 2011). We computed correlations between the wavelet coefficients of a given sub-band for all ROIs identified in GLM contrasts. We then evaluated the crosscorrelation matrices between these ROIs for each experimental rest condition and for four different wavelet sub-bands covering the typical resting state frequency range $(<0.1 \mathrm{~Hz})$. Nonparametric permutation tests were used to compare the different conditions statistically.

To compute the pairwise correlation between regions, the time courses corresponding to all blocks of a specific condition (e.g., rest post regret) were extracted. Then, the discrete wavelet transform decomposed the signal into four different frequency bands: (1) $0.02-0.04 \mathrm{~Hz}$, (2) $0.04-$ $0.07 \mathrm{~Hz}$, (3) $0.07-0.14 \mathrm{~Hz}$, and (4) $0.14-0.28 \mathrm{~Hz}$. The intermediate frequency intervals ( 2 and 3 ) are known to encompass both the typical default mode band and phasic responses, so these constituted our main "bands of interest." We will therefore report results from these two bands only (the two other bands were also examined but yielded no significant results). By applying the wavelet transform, a set of wavelet coefficients was first obtained for each region separately and then correlations between the coefficients of all possible pairs of regions were calculated. Therefore, we obtained correlation matrices for each resting condition in the experiment (e.g., rest post gratification, rest post regret).

To test statistically for differences between the two resting conditions, the correlation matrices corresponding to each condition were compared by nonparametric permutation testing (Nichols and Holmes, 2002). To obtain the distribution, under the null hypothesis, of the subjectaveraged difference between the two conditions, the condition labels of the correlation matrices were randomly permuted many times. A total of 5000 relabelings gave a reliable estimate of the rejection probability of our measure of interest. The $p$ value of the difference between conditions obtained for the correlation between each pair of regions was then converted into an equivalent $z$-score. Significant correlations with $z>2$ are marked by a single asterisk (equivalent to $p<0.01$, one-tailed, uncorrected) and those with $z>2.50$ by a double asterisk (equivalent to $p<$ 0.003 , one-tailed, uncorrected).

\section{Results}

To keep a natural flow during scanning and to avoid responseinduced biases, we did not acquire self-reports of subjective emotion experience during fMRI. We first validated our paradigm in a separate behavioral study where volunteers $(N=11$, none included in the fMRI study) were asked to rate how satisfied they felt (from -5 not at all, to +5 very much) after each streaks of 4 favorable or unfavorable gamble turns. Satisfaction ratings were positive (mean 3.15) when gains were greater than rejected alternatives (streak levels $1-3$ ), and negative (mean -3.17) when smaller (levels 4-6). A $2 \times 2$ ANOVA confirmed a significant main effect of actual gain (high vs low, $F_{(2,10)}=224, p<0.0001$ ), but, more critically, an interaction with alternative gain ( 3 different levels in each case, $\left.F_{(2,20)}=3.60, p<0.046\right)$. Because the average actual gain was comparable across the three different levels in the high and low conditions, these results show that the subjective satisfaction experienced by participants in our task was mainly driven by the difference between the actual gain and the rejected alternatives. This pattern is the hallmark of counterfactual thinking associated with regret and gratification (Mellers et al., 1999; Camille et al., 2004; Coricelli et al., 2005; Connolly and Butler, 2006). Post hoc one-tailed $t$ tests also revealed that emotion ratings on level 6 (L6) were significantly lower than those on L4 $(p<0.008)$, whereas the difference between the ratings on L1 


\section{Game period (during + /- outcomes)}

A Gratification PM / game
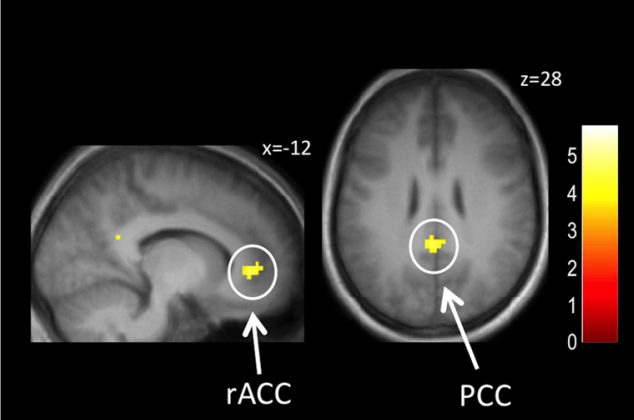

B Regret PM / game

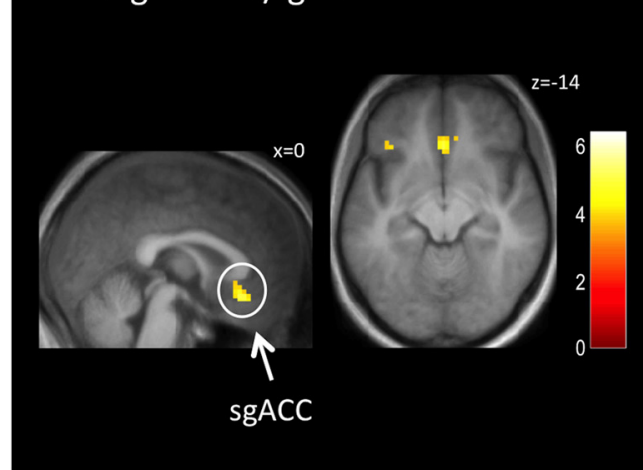

\section{Resting state period (after $+/$ - outcomes)}

\section{Gratification PM / REST}

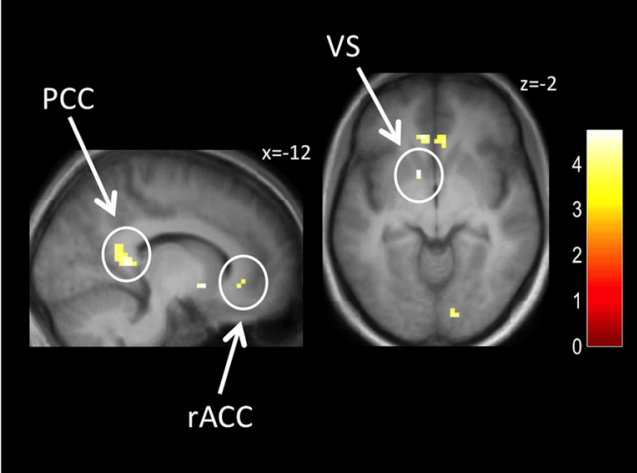

D Regret PM / REST

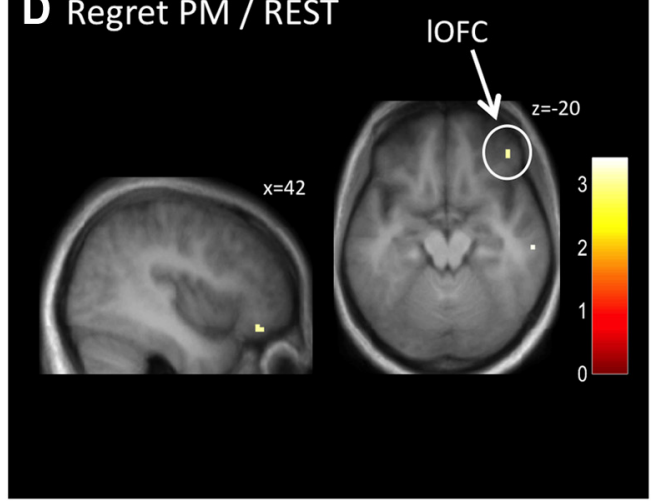

Figure 3. Brain regions showing parametric responses to increasing gratification levels $(\boldsymbol{A})$ or increasing regret levels $(\boldsymbol{B})$ during outcome presentation in the game blocks, highlighting rostral ACC and PCC for gratification and subgenual $A C C$ and posterior IOFC for regret. Parametric activations during the subsequent rest period were found for increasing gratification in rostral $A C C, P C C$, and VS $(\boldsymbol{C})$ and for increasing regret in right anterior I0FC only (D). All effects are at $p<0.001$ uncorrected, except $\boldsymbol{D}$, where $p<0.005$ (for illustration purposes). PM, Parametric modulation.

and L3 showed a trend toward significance $(p<0.067)$. Therefore, our paradigm produced a consistent modulation of outcome ratings by the unobtained gains, which is consistent with our predictions and counterfactual processing.

\section{Parametric fMRI analysis}

In participants undergoing fMRI $(n=18)$, we first identified brain regions with a response to positive or negative outcomes that increased proportionally to increasing levels of gratification or regret (using a parametric regression analysis of the difference between gains associated with actual choice and alternatives).

For the game blocks, we found significant gratification effects in the rostral part of anterior cingulate cortex (rACC), which showed increasing activation when favorable outcomes (high gains) were associated with an increasing difference relative to rejected alternatives (streak levels $1>2>3$ ). The left inferior frontal gyrus (IFG), left anterior-lateral orbitofrontal cortex (OFC), dorsomedial prefrontal cortex (dmPFC), precuneus, and posterior cingulate cortex (PCC) also showed the same parametric response (Fig. $3 A$, Table 1). Conversely, the subgenual anterior cingulate cortex (sgACC) showed an opposite pattern, with increasing activation for unfavorable outcomes (low gains) when the difference with rejected alternatives became larger (streak levels $6>5>4$ ), consistent with a regret effect (Fig. 3B). Similar effects were observed in left/right posterior-lateral OFC, right
Table 1. Brain regions showing parametric activation during the game period, in proportion to increasing gratification and regret levels

\begin{tabular}{llrrrrr}
\hline Region & Side & $x$ & $y$ & $z$ & $z$-score & $\begin{array}{l}\text { Cluster } \\
\text { size }\end{array}$ \\
\hline Gratification parametric effects & & & & & & \\
IFG & $\mathrm{L}$ & -48 & 29 & 4 & 4.20 & 4 \\
rACC & $\mathrm{L}$ & -9 & 32 & 1 & 3.72 & 19 \\
PCC & & 0 & -46 & 31 & 3.69 & 34 \\
Prec & $\mathrm{L}$ & -12 & -52 & 22 & 3.17 & \\
Cereb & $\mathrm{R}$ & 27 & -61 & -23 & 3.60 & 10 \\
rACC & $\mathrm{R}$ & 6 & 32 & -2 & 3.47 & 5 \\
IOFC & $\mathrm{L}$ & -33 & 35 & -14 & 3.39 & 11 \\
dmPFC & $\mathrm{L}$ & -9 & 62 & 19 & 3.38 & 5 \\
MCCC & & 0 & -16 & 37 & 3.15 & 2 \\
Regret parametric effects & & & & & & \\
SMG & $\mathrm{L}$ & -63 & -43 & 34 & 4.47 & 39 \\
SgACC & & 0 & 26 & -14 & 3.83 & 47 \\
SgACC & $\mathrm{L}$ & -3 & 23 & -5 & 3.31 & \\
IOFC & $\mathrm{R}$ & 36 & 26 & -20 & 3.83 & 12 \\
SMA & $\mathrm{L}$ & -9 & 14 & 61 & 3.35 & 2 \\
vmPFC & $\mathrm{R}$ & 12 & 59 & -8 & 3.34 & 2 \\
IOFC & $\mathrm{L}$ & -36 & 26 & -14 & 3.30 & 5 \\
IFG & $\mathrm{R}$ & 42 & 29 & 4 & 3.27 & 4 \\
\hline
\end{tabular}

Prec, Precuneus; Cereb, cerebellum. 
Table 2. Brain regions showing parametric activation during rest periods in proportion to increasing gratification in the preceding game block

\begin{tabular}{llrrrrr}
\hline Region & Side & \multicolumn{1}{l}{$x$} & \multicolumn{1}{l}{$y$} & \multicolumn{1}{l}{$z$} & z-score & Cluster size \\
\hline VS & $\mathrm{L}$ & -12 & 8 & -8 & 3.68 & 2 \\
rACC & $\mathrm{L}$ & -9 & 32 & -8 & 3.67 & 17 \\
PCC & $\mathrm{L}$ & -15 & -46 & 7 & 3.61 & 35 \\
Prec & $\mathrm{L}$ & -12 & -52 & 19 & 3.32 & \\
Cereb & $\mathrm{L}$ & -39 & -76 & -26 & 3.44 & 23 \\
Cereb & $\mathrm{L}$ & -27 & -73 & -23 & 3.42 & \\
rACC & $\mathrm{R}$ & 9 & 26 & -14 & 3.44 & 17 \\
Insula & $\mathrm{R}$ & 33 & 14 & 10 & 3.42 & 5 \\
V1 & $\mathrm{R}$ & 12 & -91 & -8 & 3.34 & 8 \\
Mid0C & $\mathrm{L}$ & -45 & -88 & 10 & 3.29 & 2 \\
Ling & $\mathrm{R}$ & 3 & -58 & 7 & 3.22 & 3 \\
Cereb & $\mathrm{L}$ & -39 & -61 & -23 & 3.21 & 6 \\
MCC & $\mathrm{R}$ & 15 & -13 & 40 & 3.17 & 4
\end{tabular}

Prec, Precuneus; Cereb, cerebellum; V1, primary visual cortex; MidOC, middle occipital cortex; Ling, lingual gyrus.

IFG, ventromedial prefrontal cortex (vmPFC), supplementary motor area (SMA), and supramarginal gyrus (SMG; Table 1). These results agree with results from other gambling paradigms (De Martino et al., 2006; Lawrence et al., 2009).

More critically, we then tested for parametric effects of outcome types on subsequent rest periods. Ventral striatum (VS), rACC, PCC, and insula showed higher activation at rest with higher levels of gratification (Fig. $3 C$ ). Similar but weaker effects occurred in middle cingulate cortex (MCC), occipital cortex, and cerebellum (Table 2). No parametric effects of increasing regret levels at rest reached our predefined significance threshold, but a selective modulation of right lateral OFC was observed with a slightly reduced threshold $(x, y, z=42,38,-20, z$-score $=2.65$, $p=0.004$; Fig. $3 D$ ), consistent with previous findings on counterfactual regret (Coricelli et al., 2005).

This parametric $\mathrm{AMRI}$ analysis converged with our behavioral data by showing that brain responses to gains (high or low) after one's own decision are profoundly modulated by counterfactual processing of the alternative outcomes. These effects do not only arise at the time of outcomes, but persist during subsequent rest periods, indicating that gratification and regret produce a lingering impact on brain state that is proportionate to the intensity of the emotion.

\section{Relation to individual personality differences}

To assess whether personality traits predicted different activation patterns at rest, individual scores on standardized questionnaires from participants in the fMRI experiment (Table 3) were used as linear regressors for contrasts of interest comparing different conditions of gratification or regret (i.e., high vs low levels of the same emotion or opposite emotion conditions, always at rest).

Results (Table 4) revealed a negative correlation between BDI scores and activity in nucleus accumbens (NAcc), VS, medial OFC (mOFC), gyrus rectus (GR), and dmPFC during rest periods after high gratification (streak L1) relative to those after high regret (streak L6; Fig. 4A). The higher the tendency to depressive symptoms, the less these brain regions typically associated with reward processing showed sustained activity at rest after gratifying outcomes.

Conversely, activity in lateral OFC (1OFC) during rest periods after high regret (streak L6), relative to low regret (L4) was significantly correlated with rumination scores (Fig. 4B). The more participants reported ruminative thinking, the more lOFC activation was enhanced by counterfactual processing of rejected alternatives when these were better than the obtained gains. Of note, previous research
Table 3. Behavioral scores for all participants as measured by BDI and RRS questionnaires

\begin{tabular}{lcl}
\hline Participant & BDI & RRS \\
\hline 1 & 3 & 30 \\
2 & 0 & 54 \\
3 & 0 & 37 \\
4 & 1 & 43 \\
5 & 5 & 27 \\
6 & 0 & 42 \\
7 & 10 & 42 \\
8 & 0 & 22 \\
9 & 1 & 30 \\
10 & 1 & 24 \\
11 & 7 & 33 \\
12 & 2 & 34 \\
13 & 10 & 70 \\
14 & 1 & 31 \\
15 & 8 & 37 \\
16 & 8 & 39 \\
17 & 0 & 34 \\
18 & 10 & 55 \\
Mean/SD & $3.72 / 3.98$ & $38 / 11.97$ \\
\hline
\end{tabular}

showed that the rumination scale is composed of two distinct factors, brooding and reflective pondering (Treynor et al., 2003). To assess the contribution of each of these subscales to the correlation observed with lOFC, we also computed separate correlations between individual scores in these two subscales and brain activity during rest periods after high versus low regret. Our analysis revealed that this correlation was mainly driven by the reflective component. Activity in $\mathrm{OFC}$ at rest after high versus low regret was reliably higher in participants with higher reflection scores, whereas brooding scores did not show any significant correlation in this contrast (Table 4). This finding suggests that rumination processes associated with OFC are rather reflecting an adaptive strategy to cope with negative affect and reduce depressive thoughts.

\section{Functional connectivity}

To better capture the functional dynamics of resting brain activity in different conditions, we performed functional connectivity analyses focusing on ROIs selected from the parametric GLM analysis in SPM (see Parametric fMRI analysis, above). Our main goal was to determine whether positive or negative affective states triggered by the outcome of the game also modulated the functional coupling between regions that showed differential responses to gratification or regret. All ROIs used for this analysis were identified by the parametric fMRI analyses (Table 5). For unilateral foci, we selected the symmetric counterpart in the other hemisphere. In addition, we selected left and right amygdala ROIs based on a previous study in which this region was shown to be modulated by different gamble contexts (De Martino et al., 2006).

As in previous work (Richiardi et al., 2011; Eryilmaz et al., 2011), we investigated functional connectivity in four wavelet sub-bands. The two extreme sub-bands $(<0.04$ and $>0.14 \mathrm{~Hz})$ did not show any reliable effects (probably due to lower signal-to-noise ratio); therefore, we report results from the two intermediate-frequency sub-bands $(0.04-0.07$ and $0.07-0.14 \mathrm{~Hz})$ that encompass typical resting state fluctuations. The time course of activity in each ROI was extracted from each rest period to compute the wavelet correlation between all pairs of regions. Rest periods from different gratification or regret conditions were then compared using nonparametric permutation tests (see Materials and Methods). 
Table 4. Regions with activity that significantly correlated with personality scores during conditions of interest

\begin{tabular}{|c|c|c|c|c|c|c|}
\hline Region & Side & $x$ & $y$ & $z$ & $z$-score & Cluster size \\
\hline \multicolumn{7}{|c|}{ BDI (-) at rest post high gratification $>$ rest post high regret } \\
\hline NAcc & $\mathrm{L}$ & -3 & 11 & -8 & 3.99 & 125 \\
\hline VS & R & 9 & 5 & -14 & 3.83 & \\
\hline $\mathrm{mOFC}$ & $\mathrm{R}$ & 18 & 32 & -20 & 3.71 & \\
\hline GR & $\mathrm{R}$ & 3 & 38 & -26 & 3.55 & 3 \\
\hline \multicolumn{7}{|c|}{ RRS at rest post high regret $>$ rest post low regret } \\
\hline IOFC & R & 24 & 35 & -17 & 3.97 & 10 \\
\hline \multicolumn{7}{|c|}{ Reflective pondering at rest post high regret $>$ rest post low regret } \\
\hline IOFC & R & 24 & 35 & -17 & 3.74 & 8 \\
\hline $\operatorname{lnf} T G$ & L & -36 & -7 & -29 & 3.39 & 1 \\
\hline \multicolumn{7}{|c|}{ Brooding at rest post high regret $>$ rest post low regret } \\
\hline- & - & - & - & - & - & - \\
\hline
\end{tabular}

Inf TG, inferior temporal gyrus; (-), negative correlation.

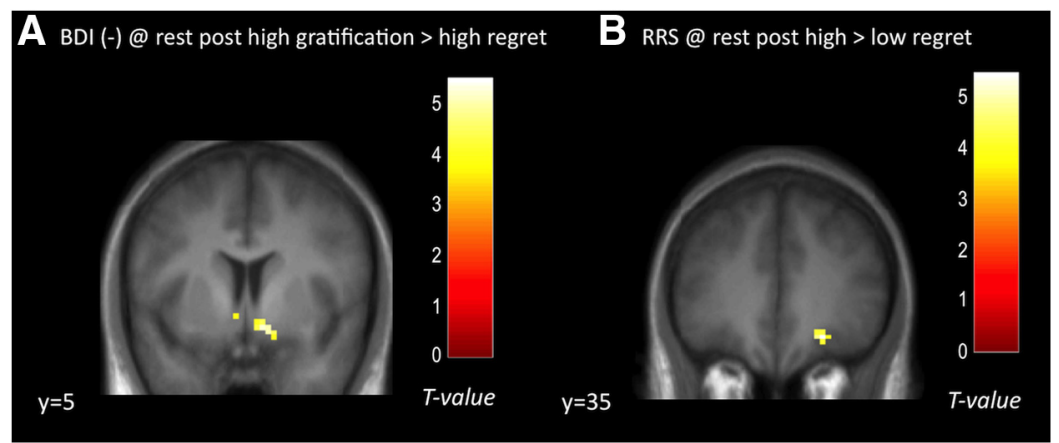

Figure 4. Correlation between individual scores on personality questionnaires and brain activity during rest conditions. BDI scores were negatively correlated with activation in the VS at rest after outcomes with the highest level of gratification (post L1) relative to outcomes with the highest level of regret (post $L 6 ; A$ ). RRS scores were positively correlated with activation in right IOFC at rest after outcomes with the highest level of regret (post L6) relative to the lowest level of regret (post $L 4 ; \boldsymbol{B})$. All effects are displayed at $p<0.001$, uncorrected.

\begin{tabular}{|c|c|c|c|c|c|}
\hline \multirow[b]{2}{*}{ Effect } & \multirow[b]{2}{*}{ Region } & \multirow[b]{2}{*}{ Side } & \multicolumn{3}{|c|}{ Coordinates } \\
\hline & & & $x$ & $y$ & $Z$ \\
\hline \multicolumn{6}{|l|}{ Game periods } \\
\hline \multirow[t]{5}{*}{ Gratification PM } & $\mathrm{rACC}$ & $L$ & -12 & 38 & -2 \\
\hline & $\mathrm{dmPFC}$ & $\mathrm{L}$ & -9 & 62 & 19 \\
\hline & $P C C$ & & 0 & -46 & 31 \\
\hline & Thalamus & $L$ & -12 & -31 & 7 \\
\hline & IFG & $\mathrm{L}$ & -48 & 29 & 4 \\
\hline Symm of left IFG & IFG & $\mathrm{R}$ & 51 & 23 & -2 \\
\hline \multirow[t]{3}{*}{ Regret PM } & IOFC & $\mathrm{L}$ & -36 & 26 & -14 \\
\hline & IOFC & $\mathrm{R}$ & 36 & 26 & -20 \\
\hline & $\operatorname{sgACC}$ & & 0 & 26 & -14 \\
\hline \multicolumn{6}{|l|}{ Rest periods } \\
\hline Postgratification PM & VS & $\mathrm{L}$ & -12 & 8 & -8 \\
\hline Symm of left VS & VS & $\mathrm{R}$ & 21 & 5 & -8 \\
\hline Postgratification PM & Insula & $\mathrm{R}$ & 33 & 14 & 10 \\
\hline Symm of right insula & Insula & $\mathrm{L}$ & -36 & 8 & 10 \\
\hline \multirow[t]{2}{*}{ De Martino et al., 2006} & Amy & $\mathrm{L}$ & -14 & 2 & -24 \\
\hline & Amy & $\mathrm{R}$ & 12 & 2 & -20 \\
\hline
\end{tabular}

PM, Parametric modulator; Symm, symmetrical Rol; Amy, amygdala.

Figure 5, $A$ and $B$, displays changes in functional connectivity for rest periods after all favorable (streak levels L1-L3) versus all unfavorable (streak levels L4-L6) outcomes. In the slower frequency sub-band $(0.04-0.07 \mathrm{~Hz})$, connectivity between sgACC and IOFC was selectively enhanced after unfavorable outcomes (Fig. 5A). The faster sub-band $(0.07-0.14 \mathrm{~Hz})$ revealed enhanced connectivity after favorable outcomes for the VS with PCC, but also for the left amygdala with PCC, as well as lOFC and dmPFC (Fig. 5B). The same region of sgACC also showed parametric activation to increasing regret levels during the game, whereas the PCC and VS responded to increasing gratification during the game and subsequent rest, respectively (see Parametric fMRI analysis, above).

Figure 5, $C$ and $D$, shows the comparison between rest periods after the most gratifying outcomes (streak level 1) and those after the most regretful outcomes (streak level 6), for the two frequency subbands. In both sub-bands, functional coupling was again selectively enhanced between PCC and VS after highly gratifying outcomes (post L1). In addition, in the same condition, the left insula was also more strongly coupled to left IFG in both sub-bands and to right lOFC in the slower subband. In the faster sub-band, the right amygdala was more strongly connected with dmPFC during rest periods after gratifying outcomes (post L1), but more coupled with right insula after regretful outcomes (post L6).

In Figure 5, $E$ and $F$, differences in connectivity are shown between rest periods after positive and negative outcomes that elicited the least gratification (streak level 3) or the least regret (streak level 4), respectively. These two conditions are those in which the difference between the participant's gain and the average of rejected alternatives is minimal. Nevertheless, higher gains are actually obtained at level 3, whereas lower gains are obtained at level 4 . Therefore, psychologically, the two conditions are clearly distinct. Functional connectivity at rest for the slow sub-band (Fig. 5E) showed only an increase in amygdala-rACC correlation after higher gains (than rejected alternatives) with low gratification levels (post L3), compared with lower gains with low regret levels (post L4). However, conversely, there were significant increases in functional connectivity of the sgACC with PCC, lOFC, IFG, and thalamus after lower gains with low regret (rest post L4 vs post L3), suggesting that coupling within this network may primarily be sensitive to relative unfavorable outcomes regardless of the degree of counterfactual difference with alternatives. In the faster sub-band (Fig. $5 F$ ), the VS was more strongly coupled to IOFC after higher gains (post L3), but more coupled with IFG after lower gains (post L4). 
sub-band 0.04-0.07 Hz

A

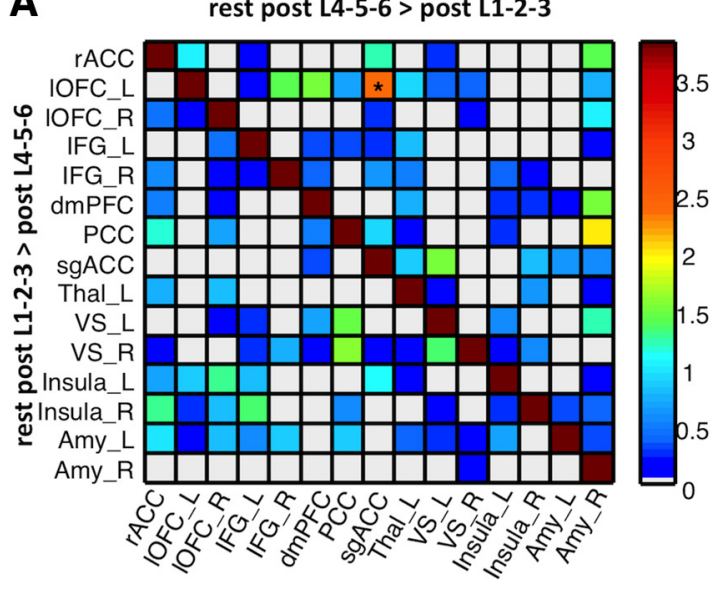

C

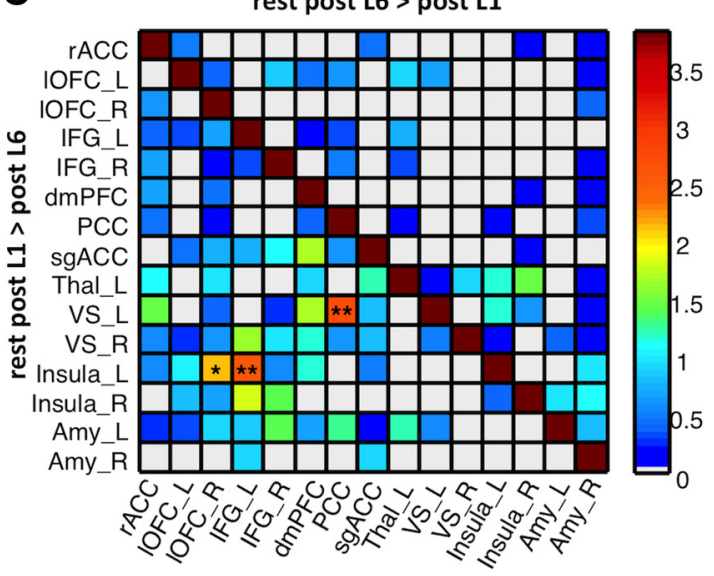

E

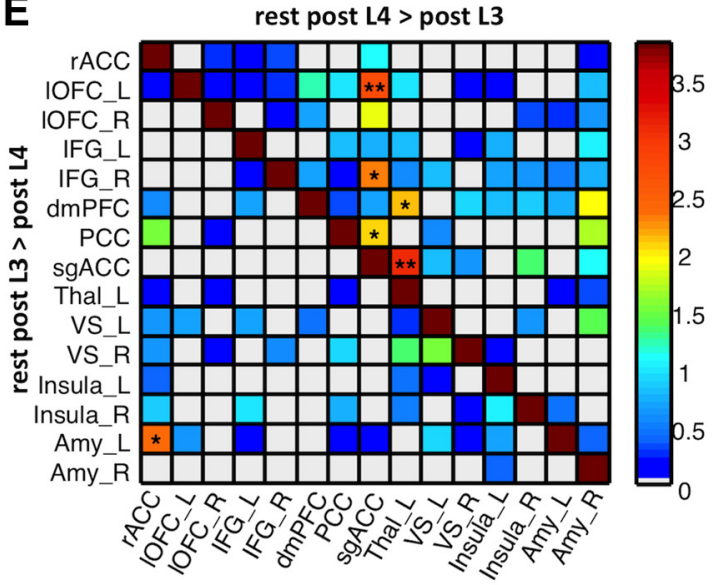

sub-band 0.07-0.14 Hz

B

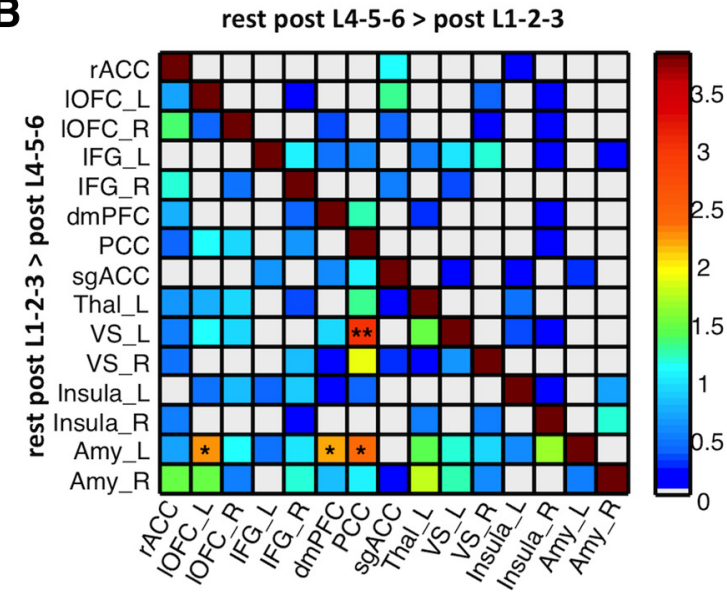

D

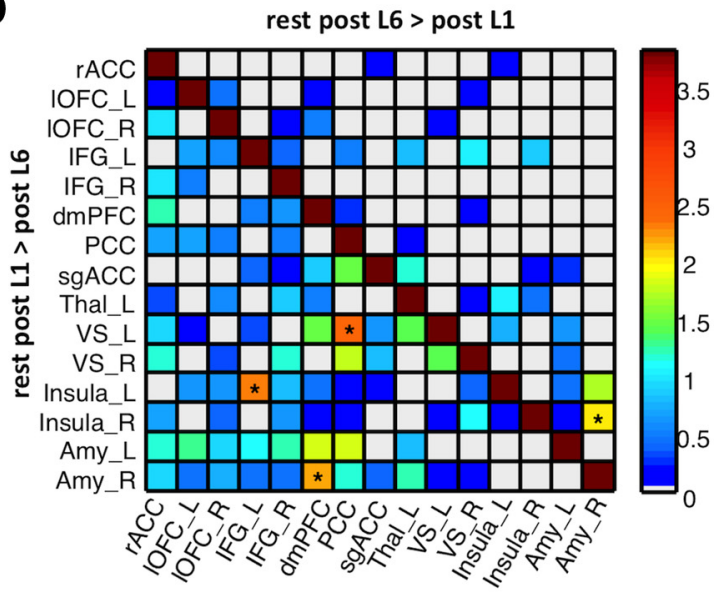

$\mathbf{F}$

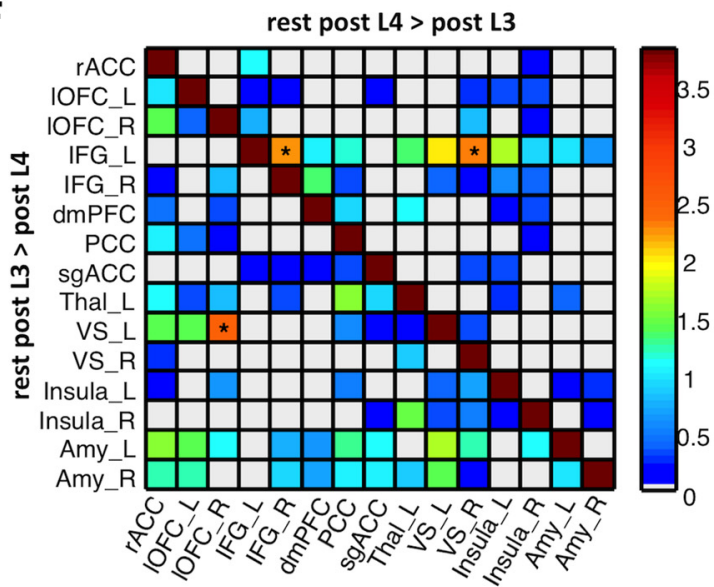

Figure 5. Changes in functional connectivity between regions of interest in rest periods after gratification and regret. These correlation matrices were computed for a lower-frequency (left) sub-band $(0.04-0.07 \mathrm{~Hz})$ and a higher-frequency (right) sub-band $(0.07-0.14 \mathrm{~Hz})$. For each matrix, the left and right halves with respect to the diagonal show the equivalent $z$-scores for opposite contrasts between two conditions (e.g., rest post gratification $>$ post regret, rest post regret $>$ post gratification). Significant changes in correlations with $z>2$ are marked by single asterisks and $z>2.5$ by double asterisks (corresponding to $p<0.01$ and $p<0.003$, one-tailed, respectively). $\boldsymbol{A}$ and $\boldsymbol{B}$ compare all levels of gratification and regret (L1, L2, L3 vs L4, L5, L6) pooled together. Contrasts are depicted in $\boldsymbol{C}$ and $\boldsymbol{D}$ for high gratification versus high regret (post L1 vs post L6) and in $\boldsymbol{E}$ and $\boldsymbol{F}$ for low gratification versus low regret (post L3 vs post L4).

Together, these data reveal modulations of distributed networks implicated in reward, feeling states, and emotion regulation, which extended during rest periods subsequent to the outcome and differed according to the nature of the outcome.

\section{Discussion}

Departing from previous research that focused on immediate brain responses to positive or negative decision outcomes (Coricelli et al., 2005; Daw et al., 2006; Chandrasekhar et al., 2008), our study investigated more sustained changes in affective state dur- 
ing rest after favorable or unfavorable outcomes of self-made choices. Our findings reveal that regret and gratification produce lingering affective states, which may subserve adaptive functions, allowing subsequent adjustment in behavior and motivation (Connolly and Butler, 2006; Summers and Duxbury, 2012). Our results also reveal that resting activity in $\mathrm{DMN}$ and related networks does not reflect fixed internal states (Raichle et al., 2001; Buckner et al., 2008), but dynamically tracks ongoing changes in affect.

Behaviorally, satisfaction ratings for gains were significantly modulated by the value of rejected alternatives, an emotional "amplification" typical of counterfactual processing associated with regret or gratification (Zeelenberg et al., 1998). In parallel, brain responses to low and high gains varied as a function of the difference with unobtained gains. During game blocks, both high and low gains activated the ACC, a region critically involved in action monitoring (Hyman et al., 2013) and affective control (Phillips et al., 2003). However, activation patterns differed for gratification and regret. The rACC responded parametrically to gratification levels after favorable outcomes, whereas the sgACC showed parametric increases to regret after unfavorable outcomes. This is consistent with previous findings of rACC response to better-than-expected outcomes (Chandrasekhar et al., 2008) as opposed to regret effects after losses in ventral prefrontal regions (Coricelli et al., 2005). In addition, rACC response to favorable outcomes was accompanied by similar parametric increases in left OFC and left IFG, whereas sgACC was accompanied by predominantly right-sided effects in OFC and IFG. Although this laterality remains to be confirmed, it is consistent with different roles for left and right hemispheres in positive and negative affect, respectively (Henriques and Davidson, 1991).

More critically, we found marked differences in brain activity during rest periods after different outcome types. Networks encompassing rACC, PCC, and VS were consistently modulated at rest after gratification. Distinct effects arose in sgACC, but also PCC, lOFC, and thalamus after regret. In addition, partly overlapping networks involving amygdala, insula, and IFG were found for both gratification and regret. These lingering emotion effects at rest were observed with both standard parametric GLM and wavelet-based analysis of functional connectivity. Furthermore, these effects were significantly modulated by personality traits, further supporting their link with subjective emotional reactivity.

Together with rACC and insula, VS showed parametric gratification effects during rest after positive outcomes. This region is crucially involved in encoding and learning reward magnitude (Daw et al., 2006; De Martino et al., 2006; Armony and Vuilleumier, 2013). Here, we show for the first time that it also exhibits sustained increases for a few minutes after a positive outcome, rather than in anticipation or reaction to rewards only. Moreover, this modulation is proportional to gratification level.

VS also showed enhanced functional coupling with PCC during rest after high gains. PCC is a key component of the DMN (Buckner et al., 2008), which may serve to link affective signals with contextual information (Nieuwenhuis et al., 2005; Vrticka et al., 2009), particularly for reward-action integration (Pearson et al., 2011). Enhanced coupling between PCC and VS may contribute to retention of information about the obtained reward and the favorable choice strategy used in the preceding game block (Hayden et al., 2008). Previous results also suggest that PCC encodes future reward magnitude and the value of past decisions to guide future actions (Pearson et al., 2011). Accordingly, neurons in PCC encode the subjective value of reward outcomes (rather than absolute value) in a way that persists across trials and predicts subsequent adjustment in behavior (Hayden et al., 2008; Pearson et al., 2011). This strong connectivity between VS and PCC and their parametric response to gratification levels could therefore mediate an appraisal process integrating reward signals with one's action and contextual information about counterfactuals, which is characteristic of self-referential emotions such as gratification or pride (Ortony et al., 1990; Connolly and Butler, 2006; Summers and Duxbury, 2012), and thus provide motivational signals for future behaviors.

Remarkably, the effect of gratification on resting activity in striatum was negatively correlated with individual proneness to depressive symptoms. The greater the tendency to low mood and self-blame, the less the striatum activated in a sustained manner after the most gratifying outcomes. This agrees with findings that negative affect reduces reward-related responses (Pizzagalli et al., 2009) and that depressed patients fail to sustain activation in NAcc to positive pictures during emotion upregulation (Heller et al., 2009). Moreover, depressed or pessimistic individuals show reduced pleasure to "better-than-expected" outcomes and less use of downward ("might-have-been-worse") counterfactual strategies during emotion appraisals (Sanna et al., 1996; Markman and Miller, 2006). Such a reduction in gratification might contribute to anhedonia and self-blame in depression through reduced integration of reward signals with self-referential processes mediated by DMN activity.

In contrast, regretful outcomes produced parametric increases in lOFC during subsequent rest. Although weak, these effects concur with OFC responses to outcome delivery in our paradigm (Table 1) and others (Camille et al., 2004; Coricelli et al., 2005). Moreover, this modulation correlated with individual rumination tendencies. These findings suggest that $1 \mathrm{OFC}$ may contribute to perseverative cognition and ruminations evoked by negative events (Mellers et al., 1999; Connolly and Butler, 2006; Brosschot et al., 2010), which is consistent with previous studies relating OFC and ventrolateral PFC activity to negative mood and rumination (Ray et al., 2005). Alternatively, lOFC might mediate self-protection strategies to cope with losses and selfcriticism, consistent with recent findings for negative socialevaluative feedback (Hughes and Beer, 2013). This interpretation would also agree with a selective correlation of OFC activity at rest with the self-reflective pondering factor of rumination rather than brooding. Furthermore, sgACC was more strongly coupled with both OFC and PCC during rest after low gains relative to high gains. The sgACC thus appears as a central "hub" in brain systems responding to unfavorable outcomes during both the game period and subsequent rest. This is consistent with a general role of this region in generating negative affect (Phillips et al., 2003 ) and regulating emotional responses to stressful events (Pezawas et al., 2005).

Regret effects in sgACC, PCC, and connected regions showed weaker parametric modulation by counterfactual information compared with gratification effects in rACC, PCC, and striatum. This was apparent in both our GLM and connectivity analysis of rest periods (Fig. 5). Regret responses might be more categorical (i.e., triggered whenever gains are lower than rejected alternatives), whereas gratification could be more graded according to the magnitude of counterfactuals (i.e., weak when gains are little different from rejected alternatives). This asymmetry would agree with psychological evidence that gratification is not a true mirror of regret (Connolly and Butler, 2006) and other affective biases making people particularly aversive to losses in decision tasks (De Martino et al., 2006). 
The amygdala was also differentially modulated at rest after favorable or unfavorable outcomes. Whereas no significant effect was found with standard GLM, connectivity analysis revealed that higher gains enhanced functional coupling of left amygdala with both PCC (compared with lower gains across different counterfactual levels; Fig. 5B) and rACC (compared with low gains with small counterfactual differences; Fig. 5E). Similar to VS-PCC connectivity, this might reflect an amygdala contribution to DMN activity at rest, encoding the affective value of past outcomes and influencing future adjustment in behavior (Pearson et al., 2011). In addition, right amygdala also showed changes in functional coupling, but with different regions depending on the condition: its connectivity increased with dmPFC after high gratification levels (L1), but with right insula after high regret (L6). These effects add to the notion that the amygdala may code for both rewarding and aversive events (Bermudez and Schultz, 2010). Although amygdala and striatum connectivity with PCC might serve to register action values and alternative options, connectivity changes with insula and dmPFC might contribute to modulations of subjective feeling states associated with favorable and unfavorable outcomes.

In conclusion, using an innovative paradigm, we show sustained effects of decision-related emotions on brain circuits implicated in self-reflexive processing at rest. VS and rACC were selectively enhanced by gratification, sgACC and OFC by regret, and both sets of regions exhibited enhanced coupling with PCC, a key node in the DMN. PCC might play a key role in integrating reward information from past outcomes and actions to guide future decisions (Pearson et al., 2011). By showing that these effects vary depending on individual tendencies to depression and rumination, our findings might have important implications for understanding neural mechanisms of mood and posttraumatic stress disorder (selfblame). More generally, our results also demonstrate that DMN activity is not static, but rather is strongly modulated by current affective state. This calls for particular caution when studying resting state fMRI in different populations and different contexts given the increasing use of such approaches to study clinical conditions.

\section{References}

Armony J, Vuilleumier P, eds (2013) The Cambridge handbook of human affective neuroscience. New York: Cambridge University.

Bermudez MA, Schultz W (2010) Reward magnitude coding in primate amygdala neurons. J Neurophysiol 104:3424-3432. CrossRef Medline

Brosschot JF, Verkuil B, Thayer JF (2010) Conscious and unconscious perseverative cognition: is a large part of prolonged physiological activity due to unconscious stress? J Psychosom Res 69:407-416. CrossRef Medline

Buckner RL, Andrews-Hanna JR, Schacter DL (2008) The brain's default network: anatomy, function, and relevance to disease. Ann N Y Acad Sci 1124:1-38. CrossRef Medline

Camille N, Coricelli G, Sallet J, Pradat-Diehl P, Duhamel JR, Sirigu A (2004) The involvement of the orbitofrontal cortex in the experience of regret. Science 304:1167-1170. CrossRef Medline

Chandrasekhar PV, Capra CM, Moore S, Noussair C, Berns GS (2008) Neurobiological regret and rejoice functions for aversive outcomes. Neuroimage 39:1472-1484. CrossRef Medline

Connolly T, Butler D (2006) Regret in economic and psychological theories of choice. Behavioral Decision Making 19:139-154. CrossRef

Coricelli G, Critchley HD, Joffily M, O'Doherty JP, Sirigu A, Dolan RJ (2005) Regret and its avoidance: a neuroimaging study of choice behavior. Nat Neurosci 8:1255-1262. CrossRef Medline

Curci A, Lanciano T, Soleti E, Rimé B (2013) Negative emotional experiences arouse rumination and affect working memory capacity. Emotion 13:867-880. CrossRef Medline
Daw ND, O’Doherty JP, Dayan P, Seymour B, Dolan RJ (2006) Cortical substrates for exploratory decisions in humans. Nature 441:876-879. CrossRef Medline

De Martino B, Kumaran D, Seymour B, Dolan RJ (2006) Frames, biases, and rational decision-making in the human brain. Science 313:684-687. CrossRef Medline

El Leithy S, Brown GP, Robbins I (2006) Counterfactual thinking and posttraumatic stress reactions. J Abnorm Psychol 115:629-635. CrossRef Medline

Eryilmaz H, Van De Ville D, Schwartz S, Vuilleumier P (2011) Impact of transient emotions on functional connectivity during subsequent resting state: a wavelet correlation approach. Neuroimage 54:2481-2491. CrossRef Medline

Hayden BY, Nair AC, McCoy AN, Platt ML (2008) Posterior cingulate cortex mediates outcome-contingent allocation of behavior. Neuron 60:1925. CrossRef Medline

Heller AS, Johnstone T, Shackman AJ, Light SN, Peterson MJ, Kolden GG, Kalin NH, Davidson RJ (2009) Reduced capacity to sustain positive emotion in major depression reflects diminished maintenance of frontostriatal brain activation. Proc Natl Acad Sci U S A 106:22445-22450. CrossRef Medline

Henriques JB, Davidson RJ (1991) Left frontal hypoactivation in depression. J Abnorm Psychol 100:535-545. CrossRef Medline

Hughes BL, Beer JS (2013) Protecting the self: the effect of social-evaluative threat on neural representations of self. J Cogn Neurosci 25:613-622. CrossRef Medline

Hyman JM, Whitman J, Emberly E, Woodward TS, Seamans JK (2013) Action and outcome activity state patterns in the anterior cingulate cortex. Cereb Cortex 23:1257-1268. CrossRef Medline

Lawrence NS, Jollant F, O’Daly O, Zelaya F, Phillips ML (2009) Distinct roles of prefrontal cortical subregions in the Iowa Gambling Task. Cereb Cortex 19:1134-1143. CrossRef Medline

Markman KD, Miller AK (2006) Depression, control, and counterfactual thinking: functional for whom? Journal of Social and Clinical Psychology 25:210-227. CrossRef

Mellers BA, Schwartz A, Ho K, Ritov I (1997) Decision affect theory: emotional reactions to the outcomes of risky options. Psychological Science 8:423-429. CrossRef

Mellers BA, Schwartz A, Ritov I (1999) Emotion-based choice. Journal of Experimental Psychology 128:332-345. CrossRef

Nichols TE, Holmes AP (2002) Nonparametric permutation tests for functional neuroimaging: a primer with examples. Hum Brain Mapp 15:1-25. CrossRef Medline

Nieuwenhuis S, Heslenfeld DJ, von Geusau NJ, Mars RB, Holroyd CB, Yeung N (2005) Activity in human reward-sensitive brain areas is strongly context dependent. Neuroimage 25:1302-1309. CrossRef Medline

Ortony A, Clore GL, Collins A (1990) The cognitive structure of emotions. Cambridge: Cambridge University.

Pearson JM, Heilbronner SR, Barack DL, Hayden BY, Platt ML (2011) Posterior cingulate cortex: adapting behavior to a changing world. Trends Cogn Sci 15:143-151. CrossRef Medline

Pezawas L, Meyer-Lindenberg A, Drabant EM, Verchinski BA, Munoz KE, Kolachana BS, Egan MF, Mattay VS, Hariri AR, Weinberger DR (2005) 5-HTTLPR polymorphism impacts human cingulate-amygdala interactions: a genetic susceptibility mechanism for depression. Nat Neurosci 8:828-834. CrossRef Medline

Phillips ML, Drevets WC, Rauch SL, Lane R (2003) Neurobiology of emotion perception I: The neural basis of normal emotion perception. Biol Psychiatry 54:504-514. CrossRef Medline

Pizzagalli DA, Holmes AJ, Dillon DG, Goetz EL, Birk JL, Bogdan R, Dougherty DD, Iosifescu DV, Rauch SL, Fava M (2009) Reduced caudate and nucleus accumbens response to rewards in unmedicated individuals with major depressive disorder. Am J Psychiatry 166:702-710. CrossRef Medline

Raichle ME, MacLeod AM, Snyder AZ, Powers WJ, Gusnard DA, Shulman GL (2001) A default mode of brain function. Proc Natl Acad Sci U S A 98:676-682. CrossRef Medline

Ray RD, Ochsner KN, Cooper JC, Robertson ER, Gabrieli JD, Gross JJ (2005) Individual differences in trait rumination and the neural systems supporting cognitive reappraisal. Cogn Affect Behav Neurosci 5:156-168. CrossRef Medline

Richiardi J, Eryilmaz H, Schwartz S, Vuilleumier P, Van De Ville D (2011) 
Decoding brain states from fMRI connectivity graphs. Neuroimage 56: 616-626. CrossRef Medline

Sanna LJ, Turley KJ, Mark MM (1996) Expected evaluation, goals, and performance mood as input. Personality and Social Psychology Bulletin 22: 323-335. CrossRef

Scherer K (2005) What are emotions? And how can they be measured? Social Science Information 44:695-729. CrossRef

Simon-Thomas ER, Godzik J, Castle E, Antonenko O, Ponz A, Kogan A, Keltner DJ (2012) An fMRI study of caring vs self-focus during induced compassion and pride. Soc Cogn Affect Neurosci 7:635-648. CrossRef Medline

Smith JM, Alloy LB (2009) A roadmap to rumination: a review of the definition, assessment, and conceptualization of this multifaceted construct. Clin Psychol Rev 29:116-128. CrossRef Medline

Summers B, Duxbury D (2012) Decision-dependent emotions and behavioral anomalies. Organizational Behavior and Human Decision Processes 118:226-238. CrossRef
Takahashi H, Matsuura M, Koeda M, Yahata N, Suhara T, Kato M, Okubo Y (2008) Brain activations during judgments of positive self-conscious emotion and positive basic emotion: pride and joy. Cereb Cortex 18:898 903. CrossRef Medline

Treynor W, Gonzalez R, Nolen-Hoeksema S (2003) Rumination reconsidered: a psychometric analysis. Cognitive Therapy and Research 27:247259. CrossRef

Vrticka P, Andersson F, Sander D, Vuilleumier P (2009) Memory for friends or foes: the social context of past encounters with faces modulates their subsequent neural traces in the brain. Soc Neurosci 4:384-401. CrossRef Medline

Wagner U, N'Diaye K, Ethofer T, Vuilleumier P (2011) Guilt-specific processing in the prefrontal cortex. Cereb Cortex 21:2461-2470. CrossRef Medline

Zeelenberg M, van Dijk WW, Manstead ASR, der Pligt J (1998) The experience of regret and disappointment. Cognition and Emotion 12:221-230. CrossRef 